\title{
Estratégias Tecnológicas de Interação e Mediação para o Ensino de Geometria Espacial: Um Estudo de Caso com Alunos Surdos
}

\author{
Cristina Paludo Santos, Luis Carlos Loose \\ Universidade Regional Integrada do Alto Uruguai e das Missões (URI) \\ 98.802-470 - Santo Ângelo - RS - Brasil \\ paludo@san.uri.br, sisloose@gmail.com
}

\begin{abstract}
This paper presents a computational solution, called GeoLibras, which establishes the link between educational software, mathematics and deaf students. The development of GeoLibras was based on several interactions with Mathematics educators and deaf students to develop strategies capable of enhancing the interaction and understanding of the didactic resources addressed by the software. The results obtained demonstrated the feasibility of the techniques used until the present moment and referred to the prospects of new research in order to increase the potential of the proposed solution.
\end{abstract}

Resumo. Este artigo apresenta uma solução computacional, denominada GeoLibras, que estabelece o elo entre software educacional, matemática e alunos surdos. $O$ desenvolvimento do GeoLibras baseou-se em diversas interações com educadores da área de Matemática e alunos surdos com vistas à elaboração de estratégias capazes de potencializar contribuições em termos de facilidade de interação e compreensão dos recursos didáticos abordados pelo software. Os resultados obtidos demonstraram a viabilidade das técnicas empregadas até o presente momento e remeteram a perspectivas de novas pesquisas com vistas a ampliar as potencialidades da solução proposta.

\section{Introdução}

A reflexão sobre a relação Tecnologia e Educação ampliou-se a partir da inclusão de alunos com deficiência na sala de aula do ensino regular. Garantir o acesso à educação a contingentes cada vez maiores da população, de forma que todos os alunos, em formação, possam estar juntos, aprendendo e participando, sem nenhum tipo de discriminação implica em repensar o processo de desenvolvimento de softwares educacionais, sobretudo, no que se refere aos recursos de interação por eles providos (Lopes, 2015).

Nesta perspectiva, a área de computação tem muito a contribuir. Em consonância com as metodologias de ensino provenientes dos estudos da área de Educação, a computação e, em especial, a área de Interface Homem-Computador (IHC), influenciada pelas novas demandas de interação, tem direcionado esforços com vistas a promover alternativas para estimular, motivar e proporcionar facilidades de interação para todos os aprendizes, inclusive os educandos com deficiência

Neste processo de inclusão destacam-se, neste trabalho, os alunos surdos. As particularidades envolvidas no processo de ensino e aprendizagem destes alunos têm sido amplamente consideradas pela comunidade de Informática na Educação. Isso pode ser 
VI Congresso Brasileiro de Informática na Educação (CBIE 2017)

Anais do XXIII Workshop de Informática na Escola (WIE 2017)

comprovado pelos inúmeros debates, teses acadêmicas e artigos que discorrem sobre este assunto. Entretanto, percebe-se que o foco maior das abordagens concentra-se em torno do bilinguismo, tecendo estratégias para potencializar o ensino e o aprendizado de Libras e da Língua Portuguesa, conforme revisões sistemáticas apresentadas por Araújo (2013) e Rocha (2014).

Embora esta questão seja altamente relevante para a comunidade surda, percebe-se uma carência de abordagens em outros contextos que fazem parte do universo desses sujeitos como, por exemplo, o aprendizado da Matemática. Neste cenário, se estabelece neste trabalho o elo entre software educacional, matemática e alunos surdos. O intuito é fazer uso dos benefícios providos pelas estratégias advindas da área de interação em prol do desenvolvimento de recursos educacionais assistivos, voltados a alunos surdos, que contemplem conteúdos da geometria espacial. Apesar de existirem softwares robustos que abordam tal conteúdo, dentre os quais citam-se o Geogebra, Régua e Compasso e Cabri, eles não esgotam as necessidades do usuário (Leite, 2007).

Os procedimentos metodológicos adotados no desenvolvimento do software denominado de GeoLibras, bem como os resultados obtidos a partir de sua disponibilização para o público-alvo, são apresentados nas seções subsequentes.

\section{Procedimentos Metodológicos}

O processo de desenvolvimento do GeoLibras baseou-se nos métodos iterativo e incremental. A partir das diversas interações com o público-alvo e com educadores da área de Matemática foi possível revisar objetivos a fim de elaborar estratégias capazes de potencializar contribuições em termos de facilidade de interação e compreensão dos recursos didáticos abordados pelo software.

O procedimento inicial compreendeu a realização de reuniões com uma educadora que atua Matemática em Libras com uma turma de alunos surdos. Tais encontros permitiram compreender as principais necessidades do grupo de alunos em relação a componentes de interação, estímulos e recursos visuais para comunicação; geraram discussões a respeito das metodologias e estratégias de ensino a serem utilizadas e, também, de conteúdos e recursos didáticos a serem contemplados no GeoLibras. Além disso, a educadora promoveu a aproximação da equipe com alunos surdos.

A reunião de fundamentos a partir de experiências e conhecimentos já produzidos, apontados por pesquisas acadêmicas, também serviu para um melhor entendimento sobre as especificidades da aprendizagem de Matemática por alunos surdos. Assim, os trabalhos desenvolvidos por Leonel (2012) com tema "o ensino de matemática para surdos inclusos em salas regulares do ensino médio: possibilidades e desafios"; Kipper (2014) que apresenta um estudo das potencialidades do ensino-aprendizagem de matemática mediado pelas TICs e, Arroio (2013) que propõe o uso de vídeos, tablet e do GeoGebra, serviram como subsídios para o desenvolvimento do GeoLibras.

Também foram realizadas pesquisas em relação aos padrões de interface e recursos de interação provenientes da área de IHC com o intuito de definir os requisitos de interação que melhor atendam às necessidades viso-espacial do público-alvo. A definição dos requisitos de interação a serem contemplados inicialmente no GeoLibras foi 
compilada a partir de pesquisas realizadas por Leite (2007) e Vaz (2012) e privilegia o uso da linguagem de sinais, ícones, textos pequenos e claros, verbos no infinitivo, mensagens de forma gráfica, animações e vídeos.

Findada a fase inicial de coleta de dados foi possível a concepção de um protótipo em baixa fidelidade que foi apresentado para a educadora e também para um grupo de alunos surdos com o intuito de realizar nova coleta de dados e, a partir dela, promover os ajustes necessários. A Figura 1 apresenta registros da avaliação inicial da ferramenta denominada GeoLibras.

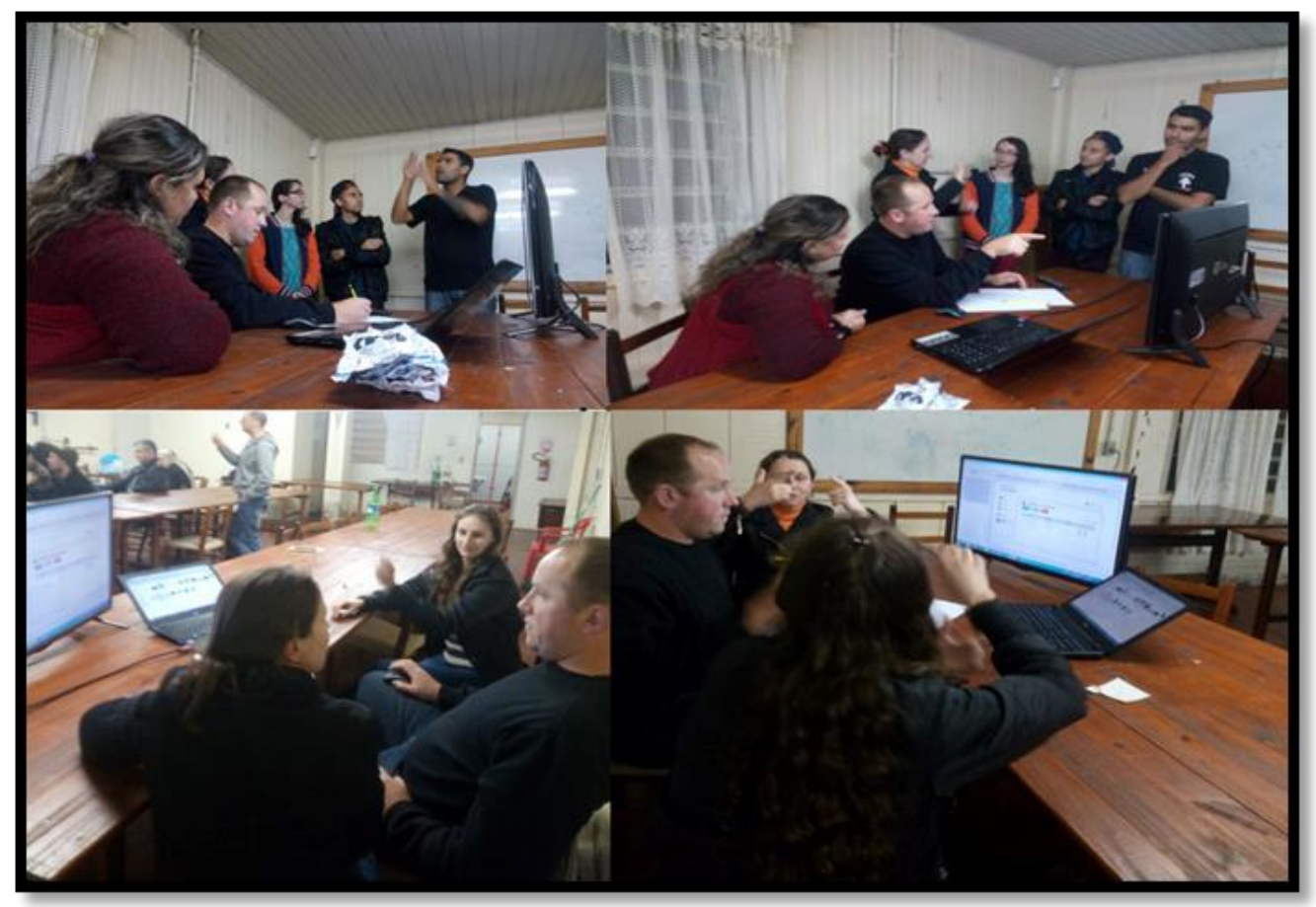

Figura 1. Avaliação inicial da ferramenta por alunos surdos e educadora

O cenário inclusivo remeteu ao aprofundamento da pesquisa e evidenciou a necessidade de incorporação de novos recursos de interação não vislumbrados na fase inicial de prototipação como, por exemplo, o uso de glosa. A glosa pode ser considerada um sistema de notação que faz uso de diversas regras para geração de um texto que possa ser traduzido para a língua de sinais de forma a manter uma estrutura sintática e semântica adequada (Ellwanger, 2015).

A pesquisa, de caráter qualitativa sob o enfoque exploratório, formou a base para o desenvolvimento de um protótipo em alta fidelidade do GeoLibras. Para o seu desenvolvimento utilizou-se o PostgreSQL para armazenamento do material pedagógico, PHP (Hypertext Preprocessor) como linguagem de programação e os frameworks API REST, Orm-doctrine, AngularJS, Bootstrap. O AngularJS torna possível gerenciar o modo como as informações das páginas serão carregadas, já, o Bootstrap oferece componentes de interface com atributos que promovem a acessibilidade e prove o sistema de grid que resulta na funcionalidade de layout responsivo. A Figura 2 apresenta a arquitetura da aplicação desenvolvida. 
VI Congresso Brasileiro de Informática na Educação (CBIE 2017)

Anais do XXIII Workshop de Informática na Escola (WIE 2017)

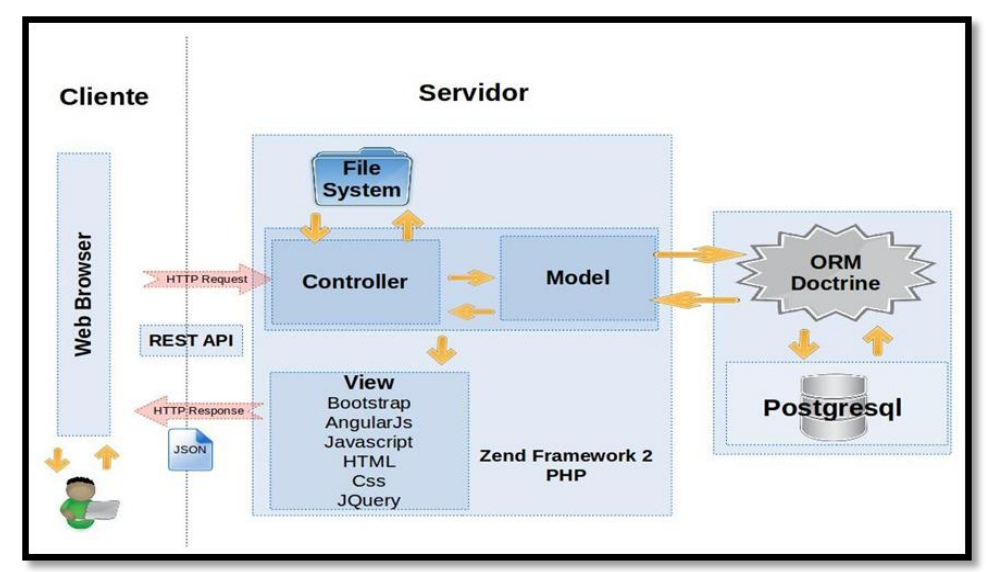

Figura 2. Arquitetura do GeoLibras

O GeoLibras apresenta funcionalidades distintas de acordo com o perfil do usuário que o acessa. Para os usuários do tipo "Professor" são disponibilizadas funções que possibilitam criar ou excluir usuários; inserir conteúdos pedagógicos que envolvam textos, glosas, imagens e vídeos; formular e disponibilizar exercícios classificados por níveis de dificuldade; criar provas; acompanhar o desempenho de uma turma ou de um aluno em específico. Além disso, a solução disponibiliza ao professor recursos de gamificação, mais especificamente de pontuação, que podem ser combinados com a solução de cada exercício proposto. Tais funcionalidades provêm robustez e dinamicidade ao GeoLibras, visto que permite ao próprio professor estruturar os recursos didáticos de maneira a reforçar suas práticas pedagógicas, além de utilizar a solução para ensinar diferentes conteúdos. A Figura 3 apresenta exemplos de interfaces para criação de conteúdos e visualização de conteúdos já existentes na base de dados.

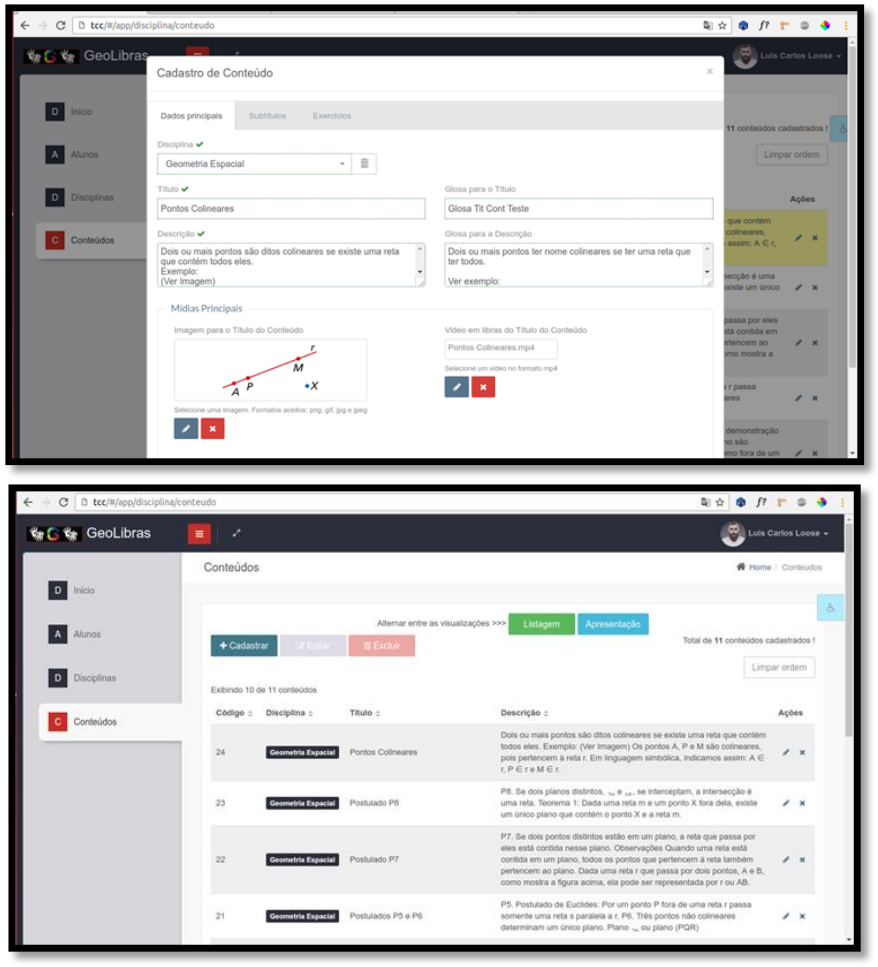

Figura 3. Interfaces do GeoLibras para o usuário "Professor" 
Já, o usuário do tipo "Aluno", tem acesso a todos os conteúdos estruturados pelo professor. Como recursos de acessibilidade disponibilizados no ambiente destacam-se vídeos em libras, glosas, aumento/redução do tamanho das fontes, tela com baixo/alto contraste, ajustes nas cores dos menus e estilos, etc., beneficiando também surdos com baixa visão. A escolha dos recursos a serem utilizados é realizada pelo próprio usuário conforme suas necessidades. A Figura 4 apresenta algumas interfaces da aplicação com os recursos de acessibilidade incorporados.

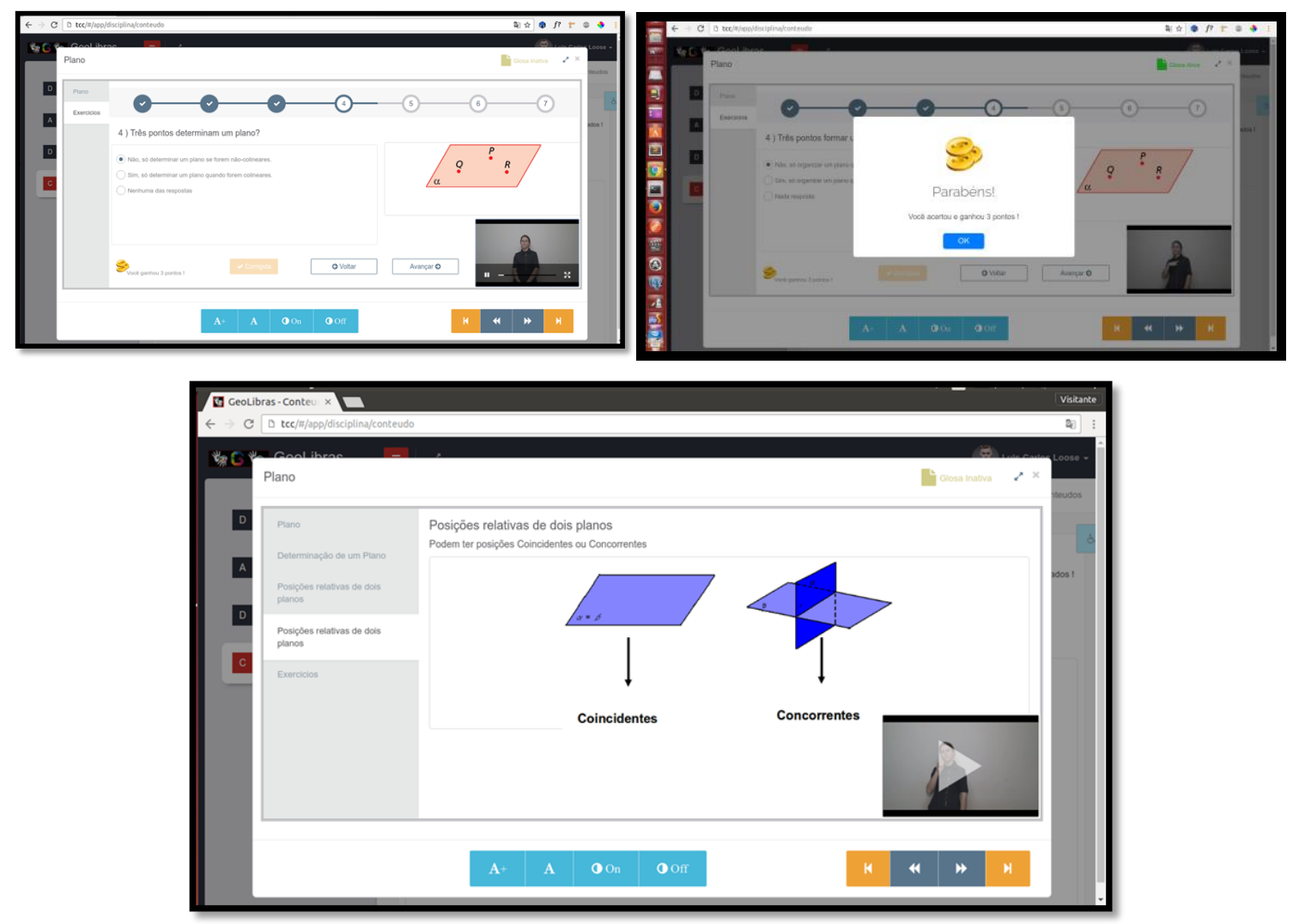

Figura 4. Interfaces do GeoLibras para o usuário "Aluno"

Embora fez-se uso do processo de avaliação formativa durante o processo de desenvolvimento do GeoLibras, uma investigação mais minuciosa é necessária a fim de verificar a adequação das estratégias de interação e mediação tecnológicas adotadas na sua concepção. Para isto realizou-se um estudo de caso em um ambiente real. Os critérios avaliativos considerados, bem como os resultados provenientes da investigação são apresentados a seguir.

\section{Resultados Obtidos}

Considerando que há uma vinculação da tecnologia com aspectos educacionais, torna-se fundamental e indispensável a realização de uma avaliação do impacto do GeoLibras sob o ponto de vista dos educadores e aprendizes. Desta maneira, um estudo de caso foi realizado para permitir a realização de uma análise que permita identificar os pontos positivos e limitações da solução proposta, tanto no que se refere aos aspectos computacionais, quanto pedagógicos. Para tanto o GeoLibras foi testado em um ambiente real envolvendo 05 alunos surdos com idade entre 18 e 23 anos, todos do ensino médio e 
VI Congresso Brasileiro de Informática na Educação (CBIE 2017)

Anais do XXIII Workshop de Informática na Escola (WIE 2017)

incluídos em escola regular, havendo variação de níveis de fluência em Libras entre intermediário e avançado. Houve também a participação de 3 professoras atuam na disciplina de Matemática.

O estudo de caso compreendeu dois momentos distintos. O primeiro deles envolveu a participação apenas das professoras e compreendeu uma breve explanação das características gerais do GeoLibras enfatizando seus objetivos e principais funcionalidades. A partir disso as professoras foram instigadas a utilizar a solução para estruturação de conteúdos didáticos. $\mathrm{O}$ desenvolvimento do conteúdo ocorreu individualmente, mas todas optaram por apresentar conceitos primitivos da Geometria Espacial, incluindo retas, pontos, planos, pontos coplanares e postulados, de acordo com o livro didático adotado pela escola.

Para coleta de dados foi utilizado um instrumento de avaliação em forma de questionário aberto constituído por 4 questões procurando oferecer um espaço para que demonstrassem pontos favoráveis e efetuassem críticas e sugestões, sem indução a respostas específicas. As questões permitem identificar: (a) se o ambiente fornece funcionalidades suficientes para criação de recursos didáticos; (b) se os recursos de acessibilidade disponíveis são adequados para atender as necessidades do público para o qual se destina; (c) quais os pontos fortes do GeoLibras e, (d) quais as limitações da solução proposta.

Os dados obtidos demonstraram aprovação parcial do GeoLibras. Embora os pressupostos educacionais tenham sido implementados a contento, duas professoras apontaram dificuldades em relação à estruturação da glosa e a disponibilização de vídeos, mas associam tal dificuldade ao pouco conhecimento sobre a notação Libras. As professoras relatam que não tiveram condições de criar recursos acessíveis sem a presença de um profissional com conhecimento em Libras. Este problema não foi enfrentado pela professora que atua Matemática em Libras. Portanto, em situações onde os professores já possuírem uma formação em Libras, os resultados poderão ser diferentes.

Dentre os pontos favoráveis apontados nos instrumentos de avaliação destacam-se a facilidade de uso; a pertinência dos recursos de acessibilidade incorporados na solução; a possibilidade de uso do GeoLibras para o ensino e aprendizagem de outros conteúdos, além dos relacionados a Geometria Espacial; a possibilidade de acompanhar a aprendizagem dos alunos através do uso de recursos como pontuação e, a forma de organização dos materiais didáticos dentro do ambiente. Uma das professoras destacou "enquanto utilizava o GeoLibras, não percebi o tempo passar, pelo fato de estar encantada com os recursos e possiblidades de acessibilidade que a aplicação aborda". A limitação relatada, apesar de não ser unânime, está atrelada à geração de glosas e vídeos em libras.

O segundo momento do estudo de caso compreendeu a disponibilização do GeoLibras, com o conteúdo criado pelas professoras, para os alunos surdos. Todos tiveram a oportunidade de utilizar e manusear o GeoLibras, sem limite de tempo estipulado ou qualquer tipo de influência.

Para coleta de dados foi utilizado um questionário composto por 9 questões fechadas e 1 aberta. As questões fechadas investigaram a usabilidade e interatividade do GeoLibras, a motivação proporcionada, a forma de apresentação, os recursos de 
VI Congresso Brasileiro de Informática na Educação (CBIE 2017)

Anais do XXIII Workshop de Informática na Escola (WIE 2017)

acessibilidade empregados, dentre outas. Já, a questão aberta permite coletar as opiniões e sugestões dos alunos. As opções de respostas para as questões fechadas consistiram em uma escala de graduação "concordo/discordo", que explicitamente rotula uma categoria e transfere o gradiente em termos de concordância versus discordância. Em cada questão, os alunos assinalaram o grau que representou sua avaliação da solução proposta em termos de "discordo totalmente", "discordo parcialmente", "não concordo nem discordo", "concordo parcialmente", e "concordo totalmente". Este tipo de resposta foi considerado pertinente à pesquisa por ressaltar as respostas extremamente positivas, negativas e irrelevantes em relação ao GeoLibras. Foram efetuadas análises qualitativas das respostas do questionário aberto e quantitativas das respostas do questionário fechado.

Por meio do questionário aberto, constatou-se que os alunos compreenderam os objetivos e procedimentos do GeoLibras, o que indica que o mesmo pode ser utilizado de acordo com as premissas iniciais estabelecidas. Dentre os comentários dos alunos que conduziram a esta afirmação citam-se: "Eu não ver nada igual Geolibras, preocupar ensinar matemática surdo, jeito certo junto Libras"; "Você clicar, conseguir perceber fácil usar aplicativo, porque mostrar claro". Tais declarações ressaltam a motivação dos estudantes ao trabalhar no GeoLibras.

Dentre as respostas obtidas a partir do questionário fechado são apresentadas as que permitiram inferências quanto às alterações necessárias e outras que comprovaram a propriedade de alguns dos elementos inicialmente projetados.

A avaliação demonstrou que o GeoLibras possui recursos que criaram expectativas e motivaram os estudantes a explorar novos conhecimentos. A realização dos desafios pedagógicos foi motivada principalmente quando o recurso de gamificação por pontos foi combinado a solução dos exercícios elaborados pelas professoras. A flexibilidade do GeoLibras quanto a sua navegação e apresentação dos conteúdos é de fundamental importância para a relação estabelecida entre o aluno e a tecnologia. Neste caso, todos os estudantes concordaram que a solução é bastante flexível a ponto ser utilizada conforme o andamento estipulado pelo estudante e sua necessidade e capacidade de assimilar e exercitar o conhecimento adquirido.

Constatou-se também que a interação que tradicionalmente ocorre entre o professor e o aluno durante as aulas de Geometria Espacial, foi modificada principalmente durante a realização dos exercícios. Por exemplo, ao invés de indagações constantes à professora para solução dos exercícios, os alunos buscavam no conteúdo disponibilizado no GeoLibras a solução para os problemas. Com isso, tornaram-se mais autônomos, realizando os exercícios no seu ritmo de trabalho, sem interferência ou direcionamento por parte da professora.

Todos os alunos concordam plenamente que os recursos de acessibilidade disponíveis são suficientes e promovem condições para a interação conforme suas necessidades. Além disso, destacaram que as interfaces estão bem organizadas permitindo que localizem com facilidade os conteúdos e as mensagens providas são compreensíveis. A partir disso, confirmou-se que a interface é intuitiva e amigável atendendo aos objetivos pré-estabelecidos. A Figura 5 apresenta registros do uso do GeoLibras por alunos surdos. 

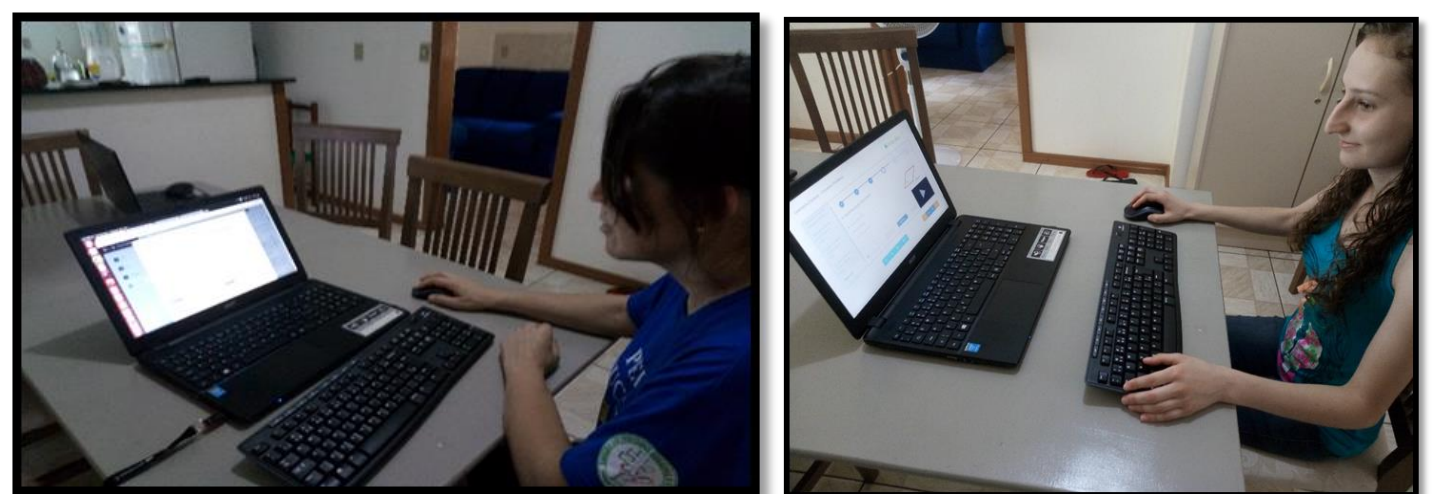

Figura 5. Avaliação do GeoLibras por usuários

O feedback do processo avaliativo permitiu vislumbrar a possibilidade de ampliação do GeoLibras contemplando novos módulos que contemplem a geração automática de glosas. A ideia é de que a partir da entrada de um texto na Língua Portuguesa o próprio GeoLibras, a partir de um módulo especialista, possa produzir as glosas. Pesquisas envolvendo estas possibilidades de ampliação da solução estão em andamento.

Tendo em vista que apenas 8 pessoas participaram do processo avaliativo, sendo 3 deles professores e 5 alunos surdos, é possível questionar o grau de generalização dos resultados. No entanto, o número reduzido de estudantes possibilitou um atendimento mais personalizado, revelando alguns fatores que somente poderiam ser verificados pela análise qualitativa. Além disso, com a amostragem considerada foi possível explorar amplamente os recursos providos pelo GeoLibras que foram considerados coerentes com o design metodológico escolhido.

Acredita-se que estudar a realidade educacional de uma determinada instituição significa tecer relações entre as situações locais concretas com as situações externas e teorias estudadas de diversos autores, podendo assim criar possibilidades para a escola pesquisada com vistas a buscar estratégias para potencializar o processo de ensino e de aprendizagem por meio do uso das tecnologias.

Cabe destacar que o GeoLibras foi disponibilizado para a escola parceira deste projeto e sua utilização ocorre em várias disciplinas. Um feedback das avaliações pedagógicas a partir do seu uso será analisado com vistas a promover constantes melhorias.

\section{Conclusões e Direcionamentos Futuros}

O desenvolvimento de um software educacional que agregue potencialidades de uso para alunos surdos não é uma tarefa trivial. Implica pensar nos saberes e práticas educacionais que perpassam o mundo contemporâneo, valorizando a articulação entre representação do conhecimento, formas de linguagem e pensamentos criativos, contribuindo para a comunicação, a interação entre pessoas e objetos de conhecimento e a aprendizagem.

Neste cenário é de suma relevância a aproximação do profissional da computação com educadores e educandos a fim de vivenciar a prática e compreender de forma mais ampla as necessidades existentes para, a partir daí, promover alternativas de interação 
VI Congresso Brasileiro de Informática na Educação (CBIE 2017)

Anais do XXIII Workshop de Informática na Escola (WIE 2017)

apropriadas e significativas que favoreçam a ambientação dos sujeitos com as novas tecnologias e supram às expectativas de saber dos alunos. A experiência vivenciada na concepção deste trabalho comprovou a importância do estabelecimento de múltiplos olhares e remeteu ao desenvolvimento do GeoLibras.

O GeoLibras foi concebido com o intuito de estabelecer alternativas de apoio ao processo de ensino e de aprendizagem de conteúdos da Matemática, visto a necessidade latente manifestada por professores e alunos. No entanto, apesar do foco inicial estar vinculado com o ensino da Geometria Espacial, seu escopo ampliou-se no decorrer das discussões a respeito das necessidades existentes, evoluindo para um ambiente que permite ao professor utilizar de seus conhecimentos pedagógicos na elaboração dos conteúdos em que o uso da tecnologia possa privilegiar e potencializar o processo de aprendizagem. Assim, o GeoLibras caracteriza-se como ambiente que possibilita a inclusão de conteúdos vinculados à qualquer disciplina de forma dinâmica, caracterizando-o como um software que possui uma aplicação prolongada ao invés de um software com conteúdos estáticos.

As atividades práticas vivenciadas durante o processo avaliativo do GeoLibras remeteram à necessidade de ampliação de recursos do ambiente, mais especificamente relacionados à geração de glosas. Assim, um módulo especialista está sendo desenvolvido e deverá ser integrado ao GeoLibras contemplando regras capazes de gerar de forma automática, a partir de um texto em português, a glosa. Este módulo permitirá que professores que desconhecem as regras para criação de glosas possam fazer uso de mais este recurso para potencializar a interação do usuário surdo com as atividades didáticas propostas.

Por fim, acredita-se que há de se somar esforços para suprir esse vazio silencioso caracterizado pela carência de tecnologias que facilite o acesso ao conhecimento pelo indivíduo surdo. Estabelecer estratégias que enriqueçam o diálogo pedagógico representa um passo à frente no que diz respeito à educação, ao desenvolvimento da criatividade e a construção do próprio sujeito, refletindo suas vivências e promovendo o aumento da autoestima, o desenvolvimento cognitivo e a autonomia destes educandos.

\section{Referências Bibliográficas}

ARAÚJO, A. L. S. O.; BRITO, R. R.; SILVA, A. P. (2013). Softwares para educação inclusiva: uma revisão sistemática no contexto de SBIE e WIE. In Brazilian Symposium on Computers in Education (Simpósio Brasileiro de Informática na Educação-SBIE) (Vol. 24, No. 1, p. 507).

ARROIO, RICHARD DOS SANTOS. "Ensino de Matemática para alunos surdos com a utilização de recursos visuais." Dissertação de Mestrado. Mestrado Profissional em Matemática em Rede Nacional. Universidade Federal Rural do Rio de Janeiro, 2013.

ELLWANGER, Cristiane; SANTOS, Cristina Paludo; SOARES, Pedro; WEISHEIMER, Tiago L. G'Libras: Um Sistema Especialista para Auxilio à Criação de Glosas. In: Anais do XXXV Congresso da Sociedade Brasileira de Computação. DESAFIE, Sociedade Brasileira de Computação: Recife, 2015. 
VI Congresso Brasileiro de Informática na Educação (CBIE 2017)

Anais do XXIII Workshop de Informática na Escola (WIE 2017)

LEITE, Maici Duarte. Design da interação de interfaces educativas para o ensino de matemática para crianças e jovens surdos. Recife, 2007. Disponível: http://www.cin.ufpe.br/ ccte/publicacoes/dissertacao_maici.pdf

LEONEL, Renata; BORGES, Fábio. "O ensino de Matemática para surdos inclusos em salas regulares do Ensino médio: possibilidades e desafios." VII Encontro de Produção Científica e Tecnológica/EPCT-Ética na Pesquisa Científica. Campo Mourão-Paraná (2012).

LOBATO, Maria José Silva. O aluno surdo e o ensino de Matemática: desafios e perspectivas na escola regular de ensino em Natal, RN. In: VI Congresso Internacional de Ensino de Matemática, 2013.

LOPES, Arilise Moraes de Almeida. "Estratégias de mediação para o ensino de matemática com objetos de aprendizagem acessíveis: um estudo de caso com alunos com deficiência visual." (2015).

KIPPER, Daiane; FRUET, Fabiane Sarmento Oliveira; KINAST, Éder Julio. Um estudo das potencialidades do ensino-aprendizagem de matemática mediado pelas tecnologias de informação e comunicação. Reflexão e Ação, v. 22, n. 1, p. 247-272, 2014.

REINOSO, Luiz; TAVARES, Orivaldo. MVLIBRAS: ambiente digital para comunidades de aprendizagem com recursos inclusivos para surdos. In: Brazilian Symposium on Computers in Education (Simpósio Brasileiro de Informática na Educação-SBIE). 2015. p. 772.

ROCHA, Denys FS; et al. "Uma Revisão Sistemática sobre a Educação do Surdo em Ambientes Virtuais Educacionais." Brazilian Symposium on Computers in Education (Simpósio Brasileiro de Informática na Educação-SBIE). Vol. 25. No. 1. 2014.

VAZ, Vagner Machado. O Uso da Tecnologia na Educação do Surdo na Escola Regular. Dissertação. Faculdade de Tecnologia de São Paulo: São Paulo, 2012.

VIEIRA, Fábia Magali Santos. Avaliação de Software Educativo: Reflexões para uma Análise Criteriosa. 2014 - Disponível em: www.edutec.net/edmagali2.htm. Último acesso: janeiro de 2017. 